\title{
CES QUARTIERS DONT ON PRÉFÉRE NE PLUS PARLER. Les métamorphoses de la politique de la ville (1977-2018)
}

\author{
Renaud Epstein (Sciences Po Saint-Germain-en-Laye, Cesdip)
}

Thomas Kirszbaum (Chercheur associé à l'Institut des Sciences sociales du Politique)

La célébration récente des quarante ans de politique de la ville nous rappelle combien cette politique s'inscrit dans la durée. Cela ne signifie pas qu'elle a suivi un développement linéaire. Au contraire, son histoire est scandée par une longue suite de réformes nationales. Invariablement, chaque émeute d'envergure ou changement de gouvernement est suivi par l'annonce d'un «plan banlieue » ou d'un train de mesures nouvelles, voire d'une loi qui prétend refonder cette politique en chantier permanent, le tout accompagné d'incessantes recompositions organisationnelles.

Cette frénésie réformatrice a construit l'image d'une politique réactive, instable et sans cap. Cette image mérite d'être sérieusement nuancée, les mesures décidées par les gouvernements successifs s'ajoutant plus souvent à celles qu'ont initiées leurs prédécesseurs qu'elles ne les effacent. Les effets de la politique de la ville étant difficiles à mesurer, les ministres qui en ont la charge - dont la longévité à ce poste est réduite ${ }^{1}$ - ont davantage intérêt à lancer de nouveaux programmes sur lesquels ils peuvent communiquer qu’à bâtir une politique de longue durée 2 . Les

\footnotetext{
${ }^{1}$ Entre 1990 et 2019, la politique de la ville a connu 20 ministres (ou secrétaires d'État), auxquels s'ajoutent 15 ministres de tutelle.

2 LE GALÈs Patrick, "Politique de la ville en France et en Grande-Bretagne:
} 
travaux néo-institutionnalistes nous rappellent que l'accumulation de mesures sédimentées est un phénomène commun à de nombreuses politiques publiques, le changement devant être considéré comme un processus progressif ${ }^{1}$. Dans le cas de la politique de la ville, la sédimentation de programmes sectoriels couvrant un nombre croissant de thématiques est une réalité qui ne doit pas faire oublier que ses principes méthodologiques ont été réaffirmés avec constance depuis le programme fondateur Habitat et vie sociale (HVS) initié en 1977 : ciblage de quartiers constituant une "géographie prioritaire»; approche globale combinant des interventions à la fois sociales, urbaines, sécuritaires et économiques; partenariat contractuel entre État et collectivités ; mobilisation des politiques dites de droit commun en faveur des quartiers; participation des habitants; évaluation régulière des programmes et des actions menées.

Cela ne signifie pas que la politique de la ville serait prisonnière des choix faits au moment de son lancement, qui l'inscriraient dans un sentier de dépendance ${ }^{2}$ dont elle ne saurait sortir. Son histoire doit plutôt se lire comme la succession de longues périodes de relative stabilité, ponctuées de rares et courtes périodes de changements radicaux. Comme théorisé par Baumgartner et Jones dans leur modèle de l'équilibre ponctué, ces réorientations surviennent lorsqu'un groupe d'acteurs parvient à imposer une image si négative de la politique considérée qu'un changement de sentier devient possible. L'approche généalogique privilégiée dans la première partie de cet article mettra en lumière ces ruptures qui jalonnent l'histoire des « plans banlieue ».

Dans une deuxième partie, ancrée dans l'actualité, on

volontarisme et ambiguités de l'État », Sociologie du travail, vol. 37, n² 2, 1995.

1 STREeCK Wolfgang et THELEN Kathleen, Beyond Continuity: Institutional Change in Advanced Political Economies, Oxford, Oxford University Press, 2005.

2 Pierson Paul, «Increasing Returns, Path Dependence, and the Study of Politics », The American Political Science Review, vol. 94, n² 2, 2000, p. 251-267.

3 Baumgartner Frank et Jones Bryan, Agenda and Instability in American Politics, Chicago, Chicago University Press, 1993. 
s'interrogera sur les évolutions récentes de la politique de la ville. Celles-ci laissent entrevoir une rupture d'une autre nature, témoignant de l'épuisement d'un long cycle d'annonces de plans d'envergure visant à convaincre l'opinion publique de la détermination de l'État à résoudre enfin une question négligée par les gouvernements précédents. Le débat sur la politique de la ville porte moins désormais sur la stratégie devant la guider que sur la légitimité même d'une telle politique. En témoigne cette déclaration du président Macron le 23 mai 2018 à l'occasion de la remise d'un rapport dont il avait passé commande à l'ancien ministre chargé de la Ville et de la Rénovation urbaine, Jean-Louis Borloo :

Je ne vais pas non plus vous annoncer un plan ville, un plan banlieue, parce que cette stratégie est aussi âgée que moi. Le premier plan de la sorte a été présenté en effet, par Raymond Barre, à peu près au moment où je naissais [...] Je crois qu'aujourd'hui, poursuivre dans cette logique c'est vouloir poursuivre dans l'assignation à résidence, la politique spécialisée, une forme de politique de clientèle en quelque sorte, qui ne bougerait pas, qui serait allouée à certains quartiers.

Tout se passe comme si l'accumulation des annonces gouvernementales au fil des décennies, mettant alternativement en scène l'octroi de ressources exceptionnelles ou de grandes réformes structurelles, avait fini par installer l'idée d'une inutilité de la politique de la ville. La perception fort répandue de «l'échec » de cette politique s'articule dorénavant avec un procès en iniquité, instruit par des entrepreneurs politiques et des procureurs médiatiques qui contestent la priorité supposément accordée par l'État à des quartiers où les immigrés et leurs descendants sont surreprésentés, auxquels ils opposent le délaissement (tout aussi présumé) d'une «France périphérique » dont les contours sont 
flous mais la couleur est claire ${ }^{1}$.

En revenant sur quatre décennies de politique de la ville, le présent article vise à rendre intelligible les métamorphoses de cette politique dont les objectifs, les cibles et les instruments révèlent des lectures évolutives du "problème des banlieues ». La mise en perspective historique permet surtout de prendre la mesure de la crise de légitimité que traverse cette politique publique autrefois surinvestie d'ambitions et désormais condamnée à la discrétion.

\section{Quarante ans de "plans banlieue" entre quartier, ville et zone}

Quartiers emblématiques des Trente glorieuses et d'un projet technocratique de modernisation de la société par l'urbain², les grands ensembles d'habitat social sont rapidement devenus les épicentres de la crise socio-urbaine française ${ }^{3}$. Depuis le programme pionnier HVS lancé en mars 1977, les "plans banlieue » se sont succédé à un rythme soutenu, avec leur cortège d'annonces plus ou moins suivies d'effets. L'impression de répétition qui s'en dégage ne doit pas masquer les changements de grande ampleur survenus à trois moments charnières, portant tout à la fois sur les orientations stratégiques et l'organisation institutionnelle de la politique de la ville. Ces tournants coïncident

\footnotetext{
1 Voir les essais de Christophe Gulluuy: Fractures françaises, Paris, François Bourin, 2010 ; La France périphérique. Comment on a sacrifié les classes populaires, Paris, Flammarion, 2014.

2 OBLET Thierry, Gouverner la ville. Les voies urbaines de la démocratie moderne, Paris, PUF, 2005 ; CUPERS Kenny, La banlieue, un projet social: ambitions d'une politique urbaine, 19451975, Marseille, Parenthèses, 2018.

3 BAUDin Gérard et GENESTIER Philippe (dir.), Banlieues à problèmes. La construction d'un problème social et d'un thème d'action publique, Paris, La Documentation française, 2002 ; Tissot Sylvie, L'État et les quartiers. Genèse d'une catégorie de l'action publique, Paris, Seuil, 2007.
} 
avec la mise en place de la Commission nationale pour le développement social des quartiers (CNDSQ) en octobre 1981, la création d'un ministère de la Ville en décembre 1990, et l'adoption de la loi d'orientation et de programmation pour la ville et la rénovation urbaine du $1^{\text {er }}$ août 2003 (dite loi Borloo).

Avec le recul historique, trois grandes périodes décennales peuvent donc être distinguées, qui correspondent chacune à une lecture particulière du "problème des banlieues » et des solutions à lui apporter, résumée dans les trois figures du quartier, de la ville et de la zone. Cette périodisation, comme l'association des trois périodes à trois orientations stratégiques distinctes, est évidemment schématique. Elle ne prétend pas décrire de manière exhaustive une politique qui a juxtaposé, au fil de son histoire, des orientations concurrentes, déclinées et combinées de façon singulière dans chaque territoire. Mais elle permet de rendre son intelligibilité aux évolutions de la politique de la ville sur la longue durée.

\section{Le quartier comme espace de mobilisation collective}

La stratégie qui prévaut à la fin des années 1970 correspond à une démarche de valorisation des quartiers prenant appui sur leurs ressources endogènes, à commencer par le potentiel d'engagement civique de leurs résidents et d'engagement professionnel et militant des agents qui œuvrent sur le terrain.

La participation des habitants occupait une place centrale dans le projet porté par les promoteurs du programme HVS, même si elle n'a eu que de faibles traductions dans les opérations locales ${ }^{1}$. Son importance a été réaffirmée par la CNDSQ, mise en place par le gouvernement Mauroy, laquelle entendait «faire des habitants des acteurs du changement ${ }^{2} »$. Le rapport d'un groupe de

\footnotetext{
${ }^{1}$ FigeAt Dominique, Bilan des opérations "Habitat et vie sociale" menées pendant la période $d u$ VIIe Plan, Rapport du Commissariat général du Plan, 1981.

2 Dubedout Hubert, Ensemble, refaire la ville, Rapport au Premier ministre, Paris,
} 
travail de la CNDSQ sur «la présence active des habitants» résumait la philosophie de cette commission que présidait Hubert Dubedout, également maire de Grenoble :

Il serait insuffisant et erroné de n'aborder la vie sociale de ces ensembles que sous l'angle de la négativité. Le développement n'est pas seulement la lutte contre tel ou tel manque ou handicap collectif; il est aussi promotion et valorisation des potentialités détenues par la population ${ }^{1}$.

Cette logique de développement endogène des quartiers présentait quelque parenté avec les démarches nord-américaines de "développement communautaire ». Mais la politique de développement social des quartiers (DSQ) en constituait une version très édulcorée. Le DSQ a certes joué un rôle pilote dans l'avènement d'une « démocratie de proximité » à partir des années 1980. Mais celle-ci a été d'initiative municipale, dans une démarche «descendante », à l'inverse des Groupes d'action municipale ou comités de quartier des années 1960 et 1970, qui procédaient d'une mobilisation "par le bas $^{2} »$. Rapidement, les associations ont été écartées des instances partenariales mises en place par l'État et les municipalités pour piloter le développement social des quartiers, et se sont vues cantonnées dans un rôle d'opérateurs chargés de mettre en œuvre les objectifs définis dans le huis clos des institutions publiques ${ }^{3}$. À défaut d'avoir véritablement insufflé un nouvel esprit démocratique, la période du DSQ a ouvert un champ d'activités portées par des associations de quartier sollicitées pour

\footnotetext{
La Documentation française, 1983.

${ }^{1}$ CNDSQ, Rapport du groupe de travail sur la présence active des habitants, 1982.

2 Gontcharoff Georges, "Le renouveau des comités de quartier», dans Loïc Blondiaux (dir.), La démocratie locale. Représentation, Participation et espace public, Paris, PUF, 1999, p. 305-328 ; CuPpers Kenny, op. cit.

3 WARIN Philippe, «L'impasse démocratique de la politique de la ville en France », Revue suisse de science politique, vol. 3, $\mathrm{n}^{\circ}$ 3, 1997, p. 1-29.; KIRSZBAUM Thomas, «Les ambitions déçues de la démocratie urbaine dans les quartiers populaires ", dans Sabrina BrESSON (dir.), Les ambivalences de la participation citoyenne: acteurs, expertises, pouvoirs et légitimités, PU François Rabelais, à paraittre.
} 
fabriquer du « lien social » entre habitants ${ }^{1}$.

\section{La ville comme échelle de transformation}

Central dans les années 1980, le thème de la participation des habitants est passé au second plan des priorités de la politique de la ville des années 1990. Cet effacement est allé de pair avec celui de la référence au quartier, dont témoigne la création du ministère de la Ville en décembre 1990. Ce ministère devait symboliser le passage d'une "politique des quartiers» appuyée sur les énergies militantes des habitants et des professionnels à une "politique de la ville » mobilisant cette fois l'ensemble des institutions locales.

Passer du quartier à la ville ne visait pas seulement à désenclaver physiquement les quartiers. Il s'agissait surtout de changer d'échelle d'intervention pour agir sur les causes structurelles de l'exclusion socio-spatiale, dont les quartiers n'étaient que les manifestations localisées. La politique de la ville a alors été redéfinie comme une politique réformatrice devant faire levier sur les autres politiques publiques, en questionnant leurs responsabilités systémiques dans la production de cette exclusion ${ }^{2}$.

La première partie des années 1990 a été principalement consacrée à mobiliser les administrations d'un État appelé à devenir «animateur ${ }^{3} »$. Cette fonction d'animation exercée par la Délégation interministérielle à la ville au niveau central trouvait son pendant local dans la figure du sous-préfet chargé de mission pour la politique de la ville, auquel il revenait d'organiser la coordination des services déconcentrés de l'État et d'animer le dialogue avec les collectivités locales. Les limites de cette mobilisation étatique n'ont

\footnotetext{
${ }^{1}$ Genestier Philippe, «Le sortilège du quartier : quand le lieu est censé faire lien. Cadre cognitif et catégorie d'action politique », Les Annales de la recherche urbaine, $\mathrm{n}^{\circ}$ 82, 1999, p. $142-154$.

2 JAILLET Marie-Christine, «La politique de la ville, une politique incertaine », Regards sur l'actualité, nº 260, 2000, p. 29-45.

${ }^{3}$ Donzelot Jacques, EstèBe Philippe, L'État animateur. Essai sur la politique de la ville, Paris, Le Seuil, 1994.
} 
pas tardé à être pointées, tant en ce qui concerne la capacité de la politique de la ville à peser sur les grandes politiques sectorielles ${ }^{1}$ que celle des nouveaux sous-préfets à s'imposer vis-à-vis des services déconcentrés et des municipalités².

L'enjeu du changement d'échelle a été réaffirmé avec force par le gouvernement Jospin à la fin des années 1990, pour justifier la construction de pouvoirs d'agglomération puissants, à même d'articuler politiques de développement et de réparation, cela afin de conjurer les risques induits par une fragmentation croissante de la ville, de moins en moins capable de «faire société ${ }^{3}$ ». Cette perspective était celle que défendait le maire socialiste d'Orléans, Jean-Pierre Sueur, dans son rapport «Demain la ville », publié en février 1998. Elle a inspiré les trois lois territoriales adoptées en 1999-20004. Avec la loi SRU, dont l'article 55 imposait à toutes les communes la construction de logements sociaux à hauteur de $20 \%$ de leur parc, et avec la création de communautés d'agglomération disposant des compétences en matière d'aménagement de l'espace, de développement économique, d'équilibre social de l'habitat et de politique de la ville, cette dernière a bénéficié d'une dynamique institutionnelle favorable au tournant des années 2000 .

Mais le changement d'échelle escompté s'est heurté à la résistance des élus municipaux ${ }^{5}$, ainsi qu’à la discontinuité des orientations de l'État, lequel a de nouveau privilégié l'échelon

\footnotetext{
1 Damamme Dominique, Jobert Bruno, «La politique de la ville ou l'injonction contradictoire en politique », Revue française de science politique, vol. 45, n 1, 1995, p. 3-30.

2 Grémion Catherine, Mounanna Christian, Le sous-préfet à la ville, Paris, L'Harmattan, 1995.

3 MAY Nicole et al. (dir.), La ville éclatée, La Tour d'Aigues, Éditions de l'Aube, 1998 ; DONZELOT Jacques, « La nouvelle question urbaine », Esprit, $\mathrm{n}^{\circ}$ 11, 1999, p. 87-114.

${ }^{4}$ Loi d'orientation pour l'aménagement et le développement durable du territoire du 25 juin 1999, dite loi Voynet; loi sur le renforcement et la simplification de la coopération intercommunale du 12 juillet 1999, dite loi Chevènement ; loi solidarité et renouvellement urbains du 13 décembre 2000, dite loi SRU.

5 Desage Fabien, Guéranger David, La politique confisquée. Sociologie des réformes et des institutions intercommunales, Bellecombes-en-Bauges, Editions du croquant, 2011.
} 
municipal pour la conduite de la politique de la ville à partir du milieu des années 2000. Dans bien des territoires, la dichotomie entre politiques de développement et de réparation ne s'est pas estompée, les interventions de la politique de la ville restant cantonnées à l'échelle des quartiers ${ }^{1}$.

\section{La zone à normaliser}

Si elle trouve ses prémices dans le Pacte de relance pour la ville (PRV) mis en place par le gouvernement Juppé en 1996, la troisième période de la politique de la ville est d'abord associée à la loi Borloo adoptée en 2003, dont l'objectif essentiel était de changer radicalement la forme urbaine et le peuplement des grands ensembles. À cette fin, la loi a institué un programme national de rénovation urbaine (PNRU), pour lequel des budgets aussi considérables qu'inédits ont été dégagés, en même temps qu'elle a transformé l'organisation institutionnelle et l'instrumentation de la politique de la ville, recomposées suivant les canons néomanagériaux ${ }^{2}$.

Objet de critiques réitérées portant sur son illisibilité et sa présumée inefficacité, la politique de la ville a ainsi été entièrement reformatée pour se conformer à l'exigence de performance induite par la loi organique aux lois de finances (Lolf) adoptée deux ans plus tôt. La loi Borloo suivait en cela les recommandations formulées par la Cour des comptes dans un rapport particulier sur la politique de la ville publié en février 2002, qui invitait à clarifier les finalités de cette politique autour d'un objectif mesurable de «réduction progressive des écarts constatés avec les autres villes

\footnotetext{
${ }^{1}$ ESTÈBE Philippe, «La politique de la ville à la bonne échelle ? L'introuvable solidarité d'agglomération", dans Thomas KIRSZBAuM (dir.), En finir avec les banlieues? Le désenchantement de la politique de la ville, La Tour d'Aigues, Éditions de l'Aube, 2015, p. 117129.

2 Epstein Renaud, La rénovation urbaine: Démolition-reconstruction de l'État, Paris, Presses de Sciences Po, 2013.
} 
ou quartiers et de "retour au droit commun"1". Reprenant mot pour mot cette formulation, la loi du $1^{\text {er }}$ août 2003 a fixé un but unique à la politique de la ville: réduire les écarts entre les 751 zones urbaines sensibles (Zus) et le reste du territoire national. L'Observatoire national des zones urbaines sensibles, créé par cette même loi, était chargé d'en suivre annuellement l'évolution statistique.

Cette manière d'envisager les quartiers sous l'angle des écarts à une moyenne statistique avait eu un précédent avec le Pacte de relance pour la ville, censé concrétiser la promesse d'un "plan Marshall pour les banlieues » faite par Jacques Chirac durant sa campagne présidentielle de 1995 menée sur le thème de la résorption de la «fracture sociale». Le PRV distinguait trois catégories de zones - zones urbaines sensibles, zones de redynamisation urbaine et zones franches urbaines - hiérarchisées en fonction d'un Indice synthétique d'exclusion. Ce classement fournissait un principe de gradation de l'effort à consentir par la puissance publique selon l'intensité de l'exclusion ${ }^{2}$.

Avec cette catégorisation, le PRV marquait le retour de la politique de la ville à l'échelle du quartier, mais au prix d'un profond changement de conception : aux débuts de la politique de la ville, les quartiers étaient sélectionnés par les élus locaux, sans que leurs frontières soient précisément délimitées et en tenant compte aussi bien de leurs difficultés que de leurs ressources ${ }^{3}$. En délimitant des zones à partir d'une opération statistique de mesure de l'intensité de leurs difficultés, la cible de la politique de la ville devenait exclusivement négative, à l'instar de l'adjectif « sensibles »

\footnotetext{
${ }^{1}$ COUR DES COMPTES, La politique de la ville, Rapport au président de la République, Paris, Journaux officiels, 2002.

${ }^{2}$ Le PRV a été officiellement présenté comme une démarche de « discrimination positive territoriale » (voir IDRAC Francis, «Le Pacte de relance pour la ville », Regards sur l'actualité, $\mathrm{n}^{\circ} 222$, juin 1996, p. 19-34). Son principal mécanisme de «traitement préférentiel» consistait en l'instauration d'un régime d'exonérations fiscales et sociales au bénéfice des entreprises installées dans les zones franches urbaines.

${ }^{3}$ ESTÈBE Philippe, L'usage des quartiers. Action publique et géographique dans la politique de la ville (1982-1999), Paris, L'Harmattan, 2004.
} 
qui les qualifiait. En occultant leurs ressources endogènes, les quartiers étaient construits comme des problèmes dont la résolution reposerait exclusivement sur l'attraction de budgets et d'habitants venus de l'extérieur.

Faisant de la réduction des écarts entre les Zus et leur environnement l'objectif unique de la politique de la ville, la loi du $1^{\text {er }}$ août 2003 adoptait le diagnostic sous-jacent à cette catégorie territoriale : celle de quartiers définis par leurs handicaps, mesurés en termes d'écart à la norme, le seul horizon pour la politique de la ville devenant celui de leur normalisation. Tel était l'objet du PNRU, vaste programme de démolition-reconstruction ${ }^{1}$ qui devait dans un même mouvement organiser la banalisation de l'urbanisme de grands ensembles hors-norme et ramener la mixité sociale dans des quartiers " ghettoïsés ${ }^{2}$ ».

Cette assimilation des cités de banlieue à des «ghettos », ou risquant de le devenir, était apparue dès la fin des années $1960^{3}$. Le programme pionner HVS cherchait déjà à « enrayer la dégradation physique et sociale de certains "grands ensembles" qui risquent de se transformer en ghettos aux portes de nos villes ${ }^{4}$ » pour $y$ «ramener une vie sociale normale ${ }^{5}$ ». Plusieurs dispositifs subséquents (mission Banlieues 89, loi d'orientation pour la Ville, Grands projets urbains, Grands projets de ville) étaient présentés comme des instruments de lutte contre les «ghettos» et se donnaient pour horizon de faire de ces quartiers « des quartiers

\footnotetext{
${ }^{1}$ La loi Borloo fixait un objectif de 200000 logements sociaux à démolir en cinq ans, ce chiffre ayant été porté à 250000 en 2005.

2 Maurin Éric, Le ghetto français. Enquête sur le séparatisme social, Paris, Le Seuil, 2004 ; LaPeYronnie Didier, Ghetto urbain. Ségrégation, violence, pauvreté en France aujourd'bui, Paris, Robert Laffont, 2008.

3 Tellier Thibault, «De la normalisation des grands ensembles à la constitution d'une politique spécifique. La genèse de la politique de la ville », dans KIRSZBAUM Thomas (dir.), En finir avec les banlieues? op. cit., p. 49-61.

4 Circulaire du 3 mars 1977 relative au fonds d'aménagement urbain et au groupe interministériel Habitat et vie sociale.

${ }^{5}$ Circulaire du 3 mars 1977 relative au fonctionnement du groupe interministériel Habitat et vie sociale.
} 
comme les autres ». Derrière cet idéal égalitaire filtre toujours une même intention: lutter contre la concentration spatiale de populations d'origine immigrée. Déjà très présente dans le programme $\mathrm{HVS}^{1}$, cette orientation a été formulée à partir du début des années 1990 comme un enjeu décisif pour la préservation du «modèle français (ou républicain) d’intégration» considéré comme menacé par cette concentration ${ }^{2}$.

Le quinquennat de Nicolas Sarkozy a été marqué par la radicalisation de ce discours public sur les quartiers, dépeints en «zones de non droit» dans une veine qui rappelle celle des néoconservateurs américains affirmant la supériorité de la civilisation occidentale et la nécessité de la défendre face aux menaces externes, en particulier celle de l'islamisme. Transposée dans l'espace national, cette grille de lecture enjoint la politique de la ville à participer au combat pour le rétablissement de l'ordre républicain dans les «territoires perdus de la République ${ }^{3}$ ». Marqué par une vague d'attentats, le quinquennat de François Hollande a vu également le discours de son Premier ministre Manuel Valls s'aligner sur cette lecture, notamment à l'occasion du comité interministériel «Égalité et citoyenneté : la République en actes » du 6 mars 2015. Sans être officiellement présenté comme tel, ce «plan banlieue » reposait sur une combinaison de mesures en faveur de la mixité urbaine, de l'intégration, et de la promotion des valeurs républicaines en guise de réponse à l'«apartheid territorial, social, ethnique » dénoncé par M. Valls ${ }^{4}$.

Mais pour la politique de la ville, le quinquennat Hollande

\footnotetext{
1 TANTER Annick et Toubon Jean-Claude, «Mixité sociale et politique de peuplement: genèse de l'ethnicisation des opérations de réhabilitation ", Sociétés contemporaines, n⿳ 33-34, 1999, p. 59-86.

2 SimON Patrick, «La politique de la ville contre la ségrégation. Ou l'idéal d'une ville sans divisions ", Les Annales de la recherche urbaine, nº 68-69, 1995, p. 26-33.

3 L'expression «territoires perdus» est empruntée à l'ouvrage, très controversé, coordonné par BENSOUSSAN Gérard, Les territoires perdus de la République : antisémitisme, racisme et sexisme en milieu scolaire, Paris, Éditions Mille et une nuits, 2002.

${ }^{4}$ KirszBaum Thomas, «La victoire posthume du Haut Conseil à l'intégration », Diversité : revue d'actualité et de réflexion pour l'action éducative, vol. 4, n 182,2015 , p. 47-53.
} 
restera surtout comme celui de la synthèse, avec l'adoption de la loi de programmation pour la Ville et la Cohésion urbaine du 21 février 2014 (dite loi Lamy). Resituée dans une perspective temporelle longue, cette réforme relève moins de la refondation que de la synthèse historique entre les approches "quartier», «ville» et «zone» qui avaient prévalu tour à tour. Faisant l'impasse sur les contradictions entre ces approches et les lectures du problème auxquelles elles renvoient, la loi Lamy réunit dans un même texte les ambitions qui ont guidé successivement la politique de la ville depuis ses origines: encouragement des démarches participatives avec la création d'un conseil citoyen dans chaque quartier prioritaire ; territorialisation des politiques publiques dans le cadre du pilotage intercommunal de la politique de la ville; banalisation urbaine et mixité sociale par la rénovation urbaine ${ }^{1}$.

\section{Une politique délégitimée}

Le Commissariat général à l'égalité des territoires $\left(\right.$ CGET $\left.^{2}\right)$ a lancé en octobre 2017 un programme de commémorations de « 40 ans de politique de la ville ». Étalée jusqu'au printemps 2018, cette célébration poursuivait un triple objectif : valoriser les actions et acteurs de la politique de la ville, donner une image plus positive des quartiers qu'elle prend pour cible et lui «donner un nouvel élan ${ }^{3} »$. Il s'agissait surtout pour le CGET d'attirer l'attention sur

\footnotetext{
${ }^{1}$ Rebaptisée « renouvellement urbain » en référence au programme éponyme du gouvernement Jospin.

${ }^{2}$ Le CGET est un service d'administration centrale créé le 31 mars 2014 par la fusion de la délégation interministérielle à l'aménagement du territoire et à l'attractivité régionale (Datar), du Secrétariat général du comité interministériel des villes (SG-CIV) et de l'Agence nationale pour la cohésion sociale et l'égalité des chances (ACSé). Il a disparu le 1er janvier 2020, pour laisser place à une nouvelle Agence nationale de la cohésion des territoires (ANCT) réunissant le CGET, l'Agence du numérique et l'Etablissement Public national d'Aménagement et de Restructuration des Espaces Commerciaux et Artisanaux (Epareca).

${ }^{3}$ Dossier de presse «Les 40 ans de la politique de la ville. Inventons les territoires de demain », Ministère de la Cohésion des territoires, octobre 2017.
} 
des quartiers et une politique présentés comme prioritaires, mais qui le devenaient de moins en moins. Alors qu'ils avaient fait l'objet de débats et de propositions diverses pendant les campagnes présidentielles de 1995, 2002, 2007 et 2012, ils ont quasiment disparu de celle de 2017. Si bien qu’à la suite de l'élection d'Emmanuel Macron, la politique de la ville ne comptait plus de ministère dédié ${ }^{1}$. À peine installé, le gouvernement Philippe a d'ailleurs opéré d'importantes coupes dans les budgets de la politique de la ville, mais aussi du logement social et des emplois aidés sur lesquels reposaient nombre d'actions de cette politique.

Ces décisions ont provoqué un mouvement de protestation des élus et responsables associatifs de banlieue, signataires de «l'appel de Grigny ${ }^{2} »$. Emmanuel Macron y a répondu en annonçant en novembre 2017 le lancement d'une «mobilisation nationale pour les habitants des quartiers", et en confiant son orchestration à Jean-Louis Borloo, ancien ministre de la Ville demeuré très populaire auprès des élus locaux. Le programme de célébration de l'anniversaire de la politique de la ville s'est ainsi accompagné d'une intense mobilisation des maires de banlieue et des réseaux associatifs, aboutissant à la remise d'un rapport visant à relancer une politique en souffrance. Mais l'anniversaire s'est mué en enterrement lors d'une spectaculaire cérémonie élyséenne, le 23 mai 2018, mettant en scène la fin de non-recevoir présidentielle opposée aux 19 propositions du rapport Borloo ${ }^{3}$.

La «mobilisation nationale pour les habitants des quartiers » n'a pas eu lieu et la mobilisation des Gilets jaunes de l'hiver 2018 rend très improbables de nouvelles initiatives publiques d'envergure en direction des quartiers populaires, tant l'ambition

\footnotetext{
${ }^{1}$ La politique de la ville a été absorbée dans un ministère de la Cohésion des Territoires. Un poste de ministre délégué à la Ville et au Logement a néanmoins été recréé 18 mois plus tard à l'occasion du remaniement d'octobre 2018.

2 http://bleublanczebre.fr/lappel-de-grigny/.

3 BORLOO Jean-Louis, Vivre ensemble, vivre en grand la République. Pour une réconciliation nationale, avril 2018 ; EPSTEIN Renaud, «En attendant le ruissellement, quels moyens pour les quartiers populaires ?», AOC Média, 10 mai 2018.
} 
en la matière semble désormais inhibée par l'appréciation d'un risque politique : celui d'exacerber le ressentiment de la France dite "périphérique», devenue le symbole d'une nouvelle fracture territoriale. La thématique de la fracture sociale symbolisée par les "cités HLM », sur laquelle Jacques Chirac avait construit sa victoire en 1995, ne fait plus recette. Une autre ligne de fracture l'a remplacée dans le débat public, qui séparerait la France des métropoles et la France des «oubliés» et des «invisibles» présentés comme les vrais perdants de la globalisation.

Popularisée par Christophe Guilluy ${ }^{1}$, cette lecture binaire a installé l'idée suivant laquelle les habitants des quartiers prioritaires de la politique de la ville seraient indûment privilégiés, bénéficiant tout à la fois de l'accès aux ressources du développement métropolitain et d'un traitement plus favorable des pouvoirs publics, par comparaison avec les espaces ruraux, du périurbain et des villes moyennes délaissés, voire méprisés par les élites politiques. L'immense succès médiatique et politique de cette thèse, pourtant très fragile sur le plan scientifique ${ }^{2}$, a joué un rôle majeur dans le processus de délégitimation d'une politique de la ville triplement contestée dans son ciblage, ses moyens et ses effets.

\section{L'extension symbolique vers les quartiers de la « France périphérique »}

Le discours sur la fracture entre métropoles et territoires périphériques semblait avoir déjà supplanté le discours historique sur la fracture sociale, intra-urbaine, quand François Hollande a habilement tenté de les articuler durant la campagne pour l'élection présidentielle de 2012. En s'emparant de la proposition de

\footnotetext{
1 Guilluy Christophe, Fractures françaises, op. cit.; GuILLuY Christophe, La France périphérique op. cit.

2 Voir le dossier de la revue du laboratoire Espaces et SOciétés : ESO, «Les chercheurs d'ESO et la "France périphérique" ", Travaux et documents, nº 41, octobre 2016.
} 
l'Association des maires Ville \& Banlieue de créer un ministère de l'Égalité des territoires (et de la Cohésion sociale) supposé répondre simultanément aux besoins de l'ensemble des territoires «délaissés » (urbains, ruraux, ultramarins), le candidat Hollande a réussi à opérer une telle synthèse. En brandissant le slogan de l'égalité territoriale, il répondait simultanément à la demande d'égalité des habitants des quartiers populaires, frappés par cinq années de crise économique et de discours stigmatisants du président sortant, et au sentiment d'abandon des habitants des villes petites et moyennes, des territoires périurbains et du monde rural, durement touchés par les restructurations industrielles et celles des services publics. Il évitait ainsi de faire apparaitre les banlieues comme les destinataires exclusifs de la sollicitude étatique ${ }^{1}$.

La création d'un ministère du Logement et de l'Égalité des territoires, confié à Cécile Duflot, puis d'un Commissariat général à l'Égalité des territoires, ont fourni une traduction institutionnelle à ce message. Il en va de même de la révision de la liste des quartiers prioritaires bénéficiant de la politique de la ville engagée simultanément par le ministre délégué à la Ville, François Lamy. Depuis la fin des années 1980, la géographie prioritaire de la politique de la ville avait fait l'objet de critiques récurrentes dans les sphères politique, académique et administrative. Ces critiques portaient notamment sur la pertinence des critères de sélection et sur l'expansion continue du nombre de quartiers ciblés, passés de quelques dizaines à la fin des années 1970 à 2500 au milieu des années 2000, au risque de contredire la notion même de priorité territoriale $^{2}$. La réforme engagée par le ministre François Lamy visait donc à resserrer et simplifier cette géographie prioritaire en

\footnotetext{
${ }^{1}$ Donzelot Jacques, «De quelle politique "l'égalité des territoires" est-elle le nom ? ", Esprit, n 8-9, 2012, p. 6-8.

${ }^{2}$ LÉvy François, Bilan/Perspectives des contrats de développement social des quartiers, Rapport du Commissariat général du Plan, Paris, La Documentation française, 1988 ; DeLaruE JeanMarie, Banlieues en difficultés: la relégation, Paris, Syros/Alternative, 1991 ; SuEuR JeanPierre, Demain, la ville, Rapport au ministre de l'Emploi et de la solidarité, Paris, La Documentation française, 1998 ; COUR DES COMPTES, op. cit., 2002.
} 
substituant aux différents découpages préexistants (751 Zones urbaines sensibles, 2200 quartiers des Contrats urbains de cohésion sociale, 500 quartiers en rénovation urbaine) une géographie unifiée qui ne devait pas excéder 400 quartiers. Ce nouveau ciblage reposait sur un critère unique : la concentration des populations pauvres d'un point de vue monétaire. L'objectif de resserrement de la géographie prioritaire s'est cependant heurté au lobbying des maires soucieux de bénéficier des crédits de la politique de la ville: la liste finalement arrêtée en 2014 compte 1300 quartiers prioritaires soit presque le double du nombre de Zus.

On retrouve dans cette liste la quasi-totalité des quartiers « historiques » de la politique de la ville. Mais en retenant le critère de la pauvreté monétaire, la nouvelle géographie prioritaire était étendue, au moins symboliquement, à des départements ruraux tels que la Creuse ou le Gers. Derrière la réforme technique, l'essentiel était le message politique indiquant un rééquilibrage des ressources de la politique de la ville en direction de territoires éloignés des grands centres urbains. Un message que François Hollande avait explicité en août 2013, alors qu'il visitait le quartier du Garros à Auch, préfecture d'un département présenté comme «le plus rural de France » :

La politique du territoire, la politique de la ville, elle concerne tout l'espace français. [...] Une politique des quartiers, ce n'est pas une politique des métropoles. [...] Un grand enjeu pour le mandat qui est le mien, c'est de permettre à chacun de penser qu'il est à l'égal de l'autre et qu'il n'y a aucune préférence pour certains quartiers par rapport à d'autres.

\section{En finir avec un traitement préférentiel... qui n'a jamais existé}

Les propos tenus par François Hollande à Auch témoignent d'une adhésion pour le moins distante au principe de discrimination positive. Celle-ci a été définie par le Conseil d'État comme «une catégorie particulière de discriminations justifiée, 
mise en œuvre par une politique volontariste, dont l'objectif est la réduction d'une inégalité ${ }^{1} »$. Pour s'en tenir à son acception la plus triviale $^{2}$, la politique de la ville permettrait de «donner plus aux quartiers qui ont moins ».

La légitimité de cette discrimination positive, pourtant hypothétique, est de plus en plus contestée. À la dénonciation rituelle par l'ex-Front national des «milliards déversés dans les banlieues» s'ajoute désormais la critique ravageuse de la discrimination à rebours, transposant à la politique de la ville française les arguments opposés par les conservateurs américains aux politiques d'affirmation action. ${ }^{3}$. Cette critique a été popularisée par Christophe Guilluy, pour qui la discrimination positive territoriale serait «en France, le faux nez de la discrimination ethnique ${ }^{4} »$. Suivant cette analyse, le traitement préférentiel des «banlieues ethnicisées » des grandes métropoles s'exercerait au détriment des couches populaires et des classes moyennes blanches résidant dans les territoires de la «France périphérique ». Cette supposée discrimination positive apparaît d'autant plus illégitime que les habitants des quartiers prioritaires bénéficieraient déjà d'un accès privilégié aux ressources urbaines (emplois, équipements et services), dont sont privés les résidents des périphéries lointaines ${ }^{5}$. Comme le résumait crûment le titre d'un article publié par FigaroVox en avril 2018 à propos du rapport Borloo, «Le plan banlieue, c'est déshabiller la France périphérique pour habiller celle qui vit de l'autre côté du périph'».

\footnotetext{
${ }^{1}$ CONSEIL D'ÉTAT, Sur le principe d'égalité, Rapport public 1996, Coll. Études \& Documents, $\mathrm{n}^{\circ}$ 48, Paris, La Documentation française, 1997.

${ }^{2}$ CALVÈs Gwenaële, La discrimination positive, Paris, PUF, « Que sais-je ?», 2004.

${ }^{3}$ KIRSZBAum Thomas, «La discrimination positive territoriale de l'égalité des chances à la mixité urbaine », Pouvoirs, n 111, novembre 2004, p. 101-118; EPSTEIN Renaud, «Un quart de siècle de rhétorique réactionnaire : l'effet pervers de la labellisation "géographie prioritaire" ", dans Thomas AgUILERA et al. (dir), Les cartes de l'action publique, Villeneuved'Ascq, PU du Septentrion, à paraître.

${ }^{4}$ GuILluY Christophe, Fractures françaises, op. cit.

${ }^{5}$ Lorrain Dominique, «La dérive des instruments. Les indicateurs de la politique de la ville et l'action publique », Revue française de science politique, vol. 56, n 3, 2006, p. 429-456.
} 
Les annonces répétées de nouveaux plans en faveur des banlieues, assortis de budgets exceptionnels, ont pu laisser croire que les quartiers dits prioritaires bénéficiaient effectivement d'un traitement préférentiel. L'existence de ce traitement de faveur est pourtant tout sauf avérée ${ }^{1}$. Les crédits votés pour le programme 147 «politique de la ville » s'élevaient à 513 millions d'euros dans la loi de finances 2019, soit moins de 0,2 \% du budget de l'État. Sachant que près de 5 millions d'habitants résident dans les quartiers prioritaires, l'ampleur de la discrimination positive est des plus limitée. Si l'on ajoute les dotations de péréquation et les dépenses fiscales associées à la politique de la ville, ainsi que les contributions à la rénovation urbaine, l'effort budgétaire de l'État est déjà plus conséquent. Dans un rapport sur les aides aux quartiers défavorisés publié en 2009, le Comité d'évaluation et de contrôle de l'Assemblée nationale avait estimé à 3,7 milliards d'euros les budgets nationaux spécifiquement dédiés à la politique de la ville, soit moins de $1 \%$ du budget de l'État.

Les moyens de la politique de la ville (qui ont fortement diminué entre 2009 et 2018) sont donc limités et corrigent tout au plus à la marge un système d'allocation des ressources publiques structurellement défavorable aux quartiers prioritaires. Si, comme le souligne la Cour des Comptes, les instruments de suivi budgétaire ne permettent pas de mesurer précisément les ressources publiques alloués aux différents territoires et leur évolution ${ }^{2}$, de nombreuses études ont montré que les crédits spécifiques de la politique de la ville - supposés s'ajouter aux moyens de droit commun - ne suffisaient pas à compenser les inégalités de traitement résultant des autres politiques publiques ${ }^{3}$.

\footnotetext{
1 KIRSZBAUM Thomas, EPSTEIN Renaud, "Synthèse des travaux universitaires et d'évaluation de la politique de la ville ", dans Comité D'Évaluation ET DE CONTRÔLE DE L'AsSEmblée NATIONALE, Quartiers défavorisés ou ghettos inavoués: la République impuissante, Rapport d'information n 2853, Assemblée nationale, 2010.

2 COUR DES COMPTES, «La politique de la ville: un cadre rénové, des priorités à préciser », Rapport public annuel, 2016.

3 Voir par exemple Comité D’Évaluation eT DE CONTRÔle DE L'AssembléE NATIONALE, Évaluation de l'action de l'État dans l'exercice de ses missions régaliennes en Seine-Saint-
} 
Dit autrement, la discrimination positive territoriale n'existe que dans l'esprit de ceux qui la dénoncent.

\section{L'échec « indiscutable » de la politique de la ville}

En paraphrasant Emmanuel Macron, on peut remarquer que la remise en cause de la politique de la ville est aussi âgée que cette dernière. Mais le discours dominant sur «l'échec» de la politique de la ville agit aujourd'hui comme un puissant ressort de désolidarisation vis-à-vis des quartiers populaires lorsqu'il se combine avec la critique du ciblage et des moyens alloués.

Depuis l'embrasement du quartier du Mas du Taureau à Vaulx-en-Velin en octobre 1990, les émeutes sporadiques que connaissent les quartiers prioritaires de la politique de la ville, le plus souvent consécutives à des violences policières, sont immanquablement suivies par un procès intenté à cette politique, dont l'échec serait ainsi démontré. De façon tout aussi immuable, il s'ensuit l'annonce de réformes ou de mesures destinées à conjurer cet échec. Force est de constater qu'en dépit de la succession des "plans banlieue », la dynamique de paupérisation des quartiers prioritaires s'est accentuée au fil des décennies ${ }^{1}$. Dès lors, comment ne pas conclure à l'échec d'une politique qui serait à l'action publique française ce que le tonneau des Danaïdes est à la mythologie grecque?

Ce jugement d'échec de la politique de la ville est pourtant discuté par les innombrables évaluations ayant cherché à en mesurer les effets et l'impact ${ }^{2}$. Cette discussion s'organise autour de deux arguments principaux, renvoyant aux biais qu'induit la

\footnotetext{
Denis, Rapport d'information n 1014, Assemblée nationale, 2018.

${ }^{1}$ Voir les données statistiques compilées chaque année par l'Observatoire national des Zones urbaines sensibles, puis par l'Observatoire national de la politique de la ville.

2 EPSTEIN Renaud, «L'évaluation en développement? Retour sur vingt ans d'évaluation de la politique de la ville », dans Brigitte BouQuet, Ivan SAINSAUlieu, et Marcel JAEger (dir.), Les défis de l'évaluation en action sociale et médico-sociale, Paris, Dunod, 2007, p. 75-94.
} 
formulation d'un jugement national sur une politique territoriale, d'une part, aux difficultés d'imputation des évolutions mesurées dans les quartiers prioritaires, d'autre part.

S'ils ont en commun de concentrer des populations défavorisées, les quartiers prioritaires ne constituent pas une catégorie homogène. Loin de l'image unifiée forgée à partir des quartiers de la Seine-Saint-Denis, la catégorie administrative des quartiers prioritaires réunit des territoires très divers: grands ensembles périphériques ou péricentraux de métropoles dynamiques, centres paupérisés de villes moyennes en décroissance, petits quartiers isolés d'anciens bassins industriels ou miniers, territoires ultramarins ${ }^{1} \ldots$ Les dynamiques d'évolution contrastées de ces territoires hétérogènes sont occultées par les statistiques nationales portant sur des valeurs moyennes. Formuler un jugement d'échec de la politique de la ville sur la base de telles données agrégées est fallacieux d'un point de vue méthodologique, mais aussi d'un point de vue analytique, en ce que cela suggère que tous ces quartiers seraient l'objet d'une même stratégie d'intervention. Rien n'est moins vrai s'agissant d'une politique territoriale dont les enjeux, les orientations, les objectifs et les actions sont définis contractuellement par les acteurs locaux, même s'ils s'inscrivent dans le cadre d'une politique nationale. De ce fait, c'est à l'échelle de chaque ville, voire de chaque quartier, qu'il convient d'apprécier si les objectifs poursuivis ont été atteints.

On ne peut au demeurant juger de ces effets à l'aune des seules évolutions statistiques mesurées. Celles-ci ne sauraient être imputées sans précaution à la politique de la ville, et nécessitent de prendre en compte les évolutions de l'environnement socioéconomique, les effets d'autres politiques publiques et les mobilités résidentielles. Mesurer les effets propres de la politique de la ville supposerait de pouvoir déterminer quelle aurait été l'évolution des quartiers prioritaires en son absence. Cette approche de

\footnotetext{
${ }^{1}$ ChAMPION Jean-Bernard et MARPSAT Maryse, «La diversité des quartiers prioritaires : un défi pour la politique de la ville », Économie et statistique, n 294-295, 1996, p. 47-65.
} 
l'évaluation par analyse contrefactuelle a connu un développement spectaculaire dans le champ des politiques sociales ou éducatives ${ }^{1}$. Sa transposition à la politique de la ville soulève d'importants problèmes méthodologiques, relatifs au contrôle des biais de sélection et à la définition des critères et périmètres de mesure des effets. Des chercheurs s'y sont cependant risqués pour tenter d'évaluer l'impact de plusieurs programmes phares de la politique de la ville (zones franches urbaines, Programme national de rénovation urbaine) ${ }^{2}$, avec des résultats bien plus nuancés que ceux des évaluations «sauvages » produites par les discours médiaticopolitiques.

Ces tentatives de mesurer l'impact de la politique de la ville apparaissent cependant fragiles, d'autant qu'elles se concentrent sur les évolutions des territoires, sans tenir compte du parcours des habitants qui y résident. Si certains programmes cherchent effectivement, à l'image de la rénovation urbaine et des zones franches, à améliorer la situation des territoires, d'autres visent d'abord la promotion sociale de leurs habitants. Ces deux objectifs apparemment complémentaires sont en réalité souvent en tension. L'implantation d'entreprises ou la construction de logements de meilleure qualité ne bénéficie pas mécaniquement aux habitants en place. Réciproquement, les actions de formation ou d'insertion contribuant à la promotion sociale de ces habitants peuvent se traduire par une dégradation paradoxale de la situation du quartier, du fait de mobilités résidentielles socialement très sélectives. Ce sont 10 à $12 \%$ des ménages résidant dans un quartier prioritaire qui déménagent chaque année, et les catégories les moins défavorisées des quartiers sont surreprésentées parmi les sortants, qui sont remplacés par de ménages entrants globalement plus

\footnotetext{
1 Jatteau Arthur, "Les évaluations par assignation aléatoire. Apports et limites », Idées économiques et sociales, vol. 3, n 193, 2018, p. 34-41.

2 Rathelot Roland et Sillard Patrick, «Zones franches urbaines: quels effets sur l'emploi salarié et les créations d'établissements?", Économie et statistique, n 415-416, 2009, p. 81-96; GUYON Nina, Étude des effets de la rénovation urbaine sur l'évolution du bâti et du peuplement dans les quartiers ciblés entre 2004 et 2013, Rapport Sciences Po - LIEPP, 2016.
} 
pauvres que ceux qui y vivent déjà ${ }^{1}$.

On ne peut donc juger du succès ou de l'échec de la politique de la ville sur le seul fondement de l'évolution des indicateurs statistiques renseignant sur la situation sociale des quartiers prioritaires. L'échec de la politique de la ville sur ce critère pourrait être lu au contraire comme un succès de ses actions de développement social, l'ascension sociale et professionnelle des habitants qui en bénéficient se traduisant tôt ou tard par le déménagement vers un autre quartier.

La profonde crise de légitimité qui affecte aujourd'hui la politique de la ville annonce-t-elle sa disparition prochaine? L'histoire invite à la prudence : combien de fois n'a-t-on annoncé la fin de cette politique qui, telle un phœnix, renait toujours de ses cendres? Les ministres qui en ont eu la charge ont eux-mêmes nourri le mythe d'une politique de la ville temporaire, ayant vocation à disparaittre une fois sa mission accomplie, au terme d'un «plan Marshall pour les banlieues» qui aurait remis à niveau les quartiers (version de droite), ou de réformes structurantes des institutions territoriales et des politiques de droit commun qui, en les mettant à la hauteur des enjeux de l'exclusion socio-spatiale, permettraient de se dispenser à moyen terme d'une politique spécifique pour les quartiers (version de gauche) ${ }^{2}$. Force est pourtant de constater que la politique de la ville a survécu à toutes les réformes qui devaient la faire disparaitre, y compris celle qu'avait laissé entrevoir le discours de campagne d'Emmanuel Macron, d’inspiration néolibérale, qui présentait le quartier comme

\footnotetext{
${ }^{1}$ Mas Stéphanie, «Mobilité résidentielle en quartiers prioritaires de la politique de la ville ", dans ONPV, Rapport annuel de l'Observatoire national de la politique de la ville, Paris, CGET, 2017.

${ }^{2}$ KirszBaum Thomas (dir.), En finir avec les banlieues? op. cit.
} 
un obstacle à l'émancipation individuelle, c'est-à-dire à la participation de chacun à la compétition sociale et économique.

Ces approches critiques de la politique de la ville ont en commun de sous-estimer le fait que le "problème des banlieues", entendu comme la concentration spatiale de ménages pauvres appartenant souvent à des minorités ethno-raciales, est un trait permanent du fonctionnement des villes dans une société inégalitaire et inscrite dans la mondialisation. Mais la reconnaissance de ce fait parait plus indicible encore quand les quartiers populaires se voient systématiquement opposer la figure concurrente des territoires « oubliés » de la «France périphérique». La politique de la ville devient alors le bouc émissaire d'une rhétorique identitaire qui mobilise des catégories territoriales pour parler d'enjeux essentiellement ethno-raciaux.

Malgré sa délégitimation croissante, la politique de la ville semble appelée à perdurer en tant que politique spécifique pour les quartiers, aucun gouvernement ne pouvant prendre le risque d'une explosion sociale majeure dans des territoires dont près de la moitié des habitants vivent sous le seuil de pauvreté. Mais la poursuite de cette politique paraît désormais conditionnée à l'abandon d'un affichage de son caractère prioritaire pour l'État, voire de toute forme d'affichage.

Pendant près de quarante ans, les grands ensembles d'habitat social visés par la politique de la ville ont symbolisé la «fracture sociale», et bénéficié à ce titre d'une attention politique et médiatique importante. "Ces quartiers dont on parle», pour reprendre le titre d'un ouvrage collectif publié en $1997^{1}$, sont désormais des quartiers dont on préfère ne plus parler, quitte à poursuivre une politique de la ville, mais sans le dire.

\footnotetext{
${ }^{1}$ Collectif, En marge de la ville, au caur de la société : ces quartiers dont on parle, La Tour d'Aigues, Éditions de l'Aube, 1997.
} 